\title{
Ahrens, Petra, Katja Chmilewski, Sabine Lang, und Birgit Sauer (2020): Gender Equality in Politics. Implementing Party Quotas in Germany and Austria Cham: Springer. 147 Seiten. CHF 65.00
}

\author{
Agnes Blome (D)
}

Angenommen: 7. Januar 2021 / Online publiziert: 26. Januar 2021

(C) Der/die Autor(en) 2021

Obwohl sich der Großteil der etablierten politischen Parteien in Deutschland und Österreich in ihren Parteisatzungen dem Ziel der Geschlechtergerechtigkeit verschrieben hat und Quoten für die parteiinterne Kandidatinnenaufstellung eingeführt hat, stagniert der Frauenanteil in den nationalen wie regionalen Parlamenten bei etwa 30 Prozent. In Brandenburg und Thüringen wurden deshalb 2019 Paritätsgesetze verabschiedet, die die Besetzung der Kandidatenlisten von Parteien mit gleich vielen Frauen und Männern für künftige Landtagswahlen vorsehen. Im Nachbarland Österreich setzte Bundeskanzler Kurz 2017 erstmalig das Reißverschlussverfahren bei der Besetzung der Kandidatinnenliste der ÖVP für die Nationalratswahl ein.

Mit der drängenden Frage, warum die Parteien mehr als 100 Jahre nach der Einführung des Frauenwahlrechts in Deutschland und Österreich so häufig ihren Anspruch verfehlen, den Anteil gewählter weiblicher Abgeordneter entsprechend ihrer Quoten zu stellen, beschäftigt sich die 2020 erschienene Monografie von Petra Ahrens, Katja Chmilewski, Sabine Lang und Birgt Sauer. Um das Implementationsdefizit zu analysieren, etablieren die Autorinnen das Konzept des ,post-quota gender gap", der angibt, wie groß die Lücke zwischen der Quote und dem Anteil von weiblichen Abgeordneten einer Partei ist. Während die Grünen Parteien in beiden nationalen Parlamenten typischerweise ihre Quote von 50 Prozent erfüllen, verfehlen die deutschen und österreichischen sozialdemokratischen Parteien ihr Ziel (40 Prozent) in einigen Wahlen um bis zu 6 respektive 18 Prozentpunkte. In beiden Ländern gilt für die konservativen Parteien CDU/CSU und ÖVP ein Quorum von 33 Prozent, das die ÖVP kontinuierlich deutlich besser erreicht als die CDU/CSU (Kap. 5).

\footnotetext{
A. Blome $(\bowtie)$

Otto-Suhr-Institut für Politikwissenschaft, Freie Universität Berlin, Berlin, Deutschland

E-Mail: agnes.blome@fu-berlin.de
} 
Ziel der Autorinnen ist es, diese Diskrepanzen zwischen selbst gestecktem Ziel und der geringeren Anzahl der Sitze für Frauen zu erklären. Dazu nutzen sie ein Most-Similar-Systems-Forschungsdesign und analysieren vergleichend Deutschland und Österreich sowohl auf nationaler wie auch auf regionaler Ebene mit jeweils zwei Ländern. Methodisch stützen sich Ahrens et al. auf umfangreiche Dokumentenanalysen, deskriptive Statistik und Expertinnen-Interviews. Hauptaugenmerk liegt auf den unterschiedlichen Wahlsystemen - Deutschland hat ein Mischsystem aus Mehrheitsund Verhältniswahl, Österreich ein reines Verhältniswahlsystem. Wie lässt sich aber erklären, dass der Frauenanteil in Deutschland trotz der Mehrheitswahlkomponente lange Zeit höher war als in Österreich? Warum sind entgegen der in der Literatur aufgestellten Annahme einer leichteren Erfüllung von Parteiquoten in Verhältniswahlsystemen österreichische Parteien nicht kontinuierlich erfolgreich darin, ihre Quoten zu erfüllen? Die Autorinnen argumentieren, dass die Entscheidung der Parteien für oder gegen die Aufstellung von Kandidaten in Wahlkreisen und/oder auf Listen mit dem Wahlsystem interagiert und dadurch die erfolgreiche Implementation von Quoten behindert. Um dieses Problem aufzulösen, müssten Parteien entweder ihre Quoten oder das Wahlsystem ändern.

Das Buch folgt einer gut aufgebauten Struktur. Nach einer Einleitung, die außerdem die Fallauswahl und Methodik erläutert, folgt eine vergleichende Darstellung über die politischen Systeme Deutschlands und Österreichs zur Kontextualisierung der Fälle. Kapitel 3 bietet einen aufschlussreichen Überblick über die Geschichte und die weltweite Verbreitung von Frauenquoten, bevor es detailliert die Entwicklung der politischen Repräsentation und den Einfluss der freiwilligen Quoten in Deutschland und Österreich vorstellt. Der Vergleich zeigt ähnliche Entwicklungen des Frauenanteils in den beiden Ländern, der nach anfänglichem Anstieg seit Beginn der 1990er-Jahre bei etwa einem Drittel verharrt. Auf Länderebene ist die Unterrepräsentation von Frauen teils noch gravierender. Kapitel 4 widmet sich den theoretischen Ansätzen zu Implementationsdefiziten von Quoten und prüft und diskutiert deren Erklärungskraft für Deutschland und Österreich mithilfe des Interviewmaterials. Auch wenn Gründe wie das geringere Angebot, fortdauernde traditionelle Geschlechterstereotype und die mangelnde Vereinbarkeit mit familiären Pflichten unzweifelhaft ein Problem darstellen, kommen Ahrens et al. zu dem Schluss, dass sie nicht die Unterschiede zwischen den Ländern und innerhalb der Länder erklären können. In den beiden folgenden Kapiteln analysieren sie daher das Zusammenspiel zwischen Quoten und dem Wahlsystem auf Bundesebene (Kap. 5) bzw. Länderebene (Kap. 6). Während die Parteien bei der Bundestagswahl 2017 ihre Quoten mittels der Landeslisten erfüllten, machen die Autorinnen die Mehrheitswahlkomponente als wesentliches Hindernis für die fortdauernde Unterrepräsentation von Frauen aus: Die geringere Platzierung der beiden großen Parteien (aber insbesondere der CDU/CSU) von Frauen in ,,sicheren Wahlkreisen “ und der ,male incumbency effect“ führten zu niedrigeren Chancen von weiblichen Kandidaten. In Österreich führen die meist von Männern besetzten aussichtsreichen Positionen der kleinen regionalen Wahlkreise, die im Verhältniswahlsystem mit den Bundes- und Landeslisten verbunden sind, zu den geringeren Chancen der weiblichen Kandidatinnen. Hinzu kommt die Möglichkeit der Vorzugsstimme auf den ansonsten quotierten Listen, die männliche Kandidaten zu privilegieren scheint. Des Weiteren sehen die Parteien keine 
Sanktionen bei Missachtung der Quoten vor. Auch auf regionaler Ebene (Kap. 6) zeigt sich, dass die Mehrheitswahlkomponente ein Hindernis für höhere Frauenanteile darstellt. Die Wahlkreisgröße ist ein entscheidender Faktor: Je mehr Mandate in einem Wahlkreis zu gewinnen sind, desto kleiner ist der ,post-quota gender gap“.

Die Stärke des Buchs liegt in seiner Tiefe der Analysen. Der sorgfältige Fallvergleich auf Bundes- und auf Landesebene ermöglicht eine detaillierte Untersuchung der zahlreichen Faktoren, die zum Nichterreichen der parteiinternen Quoten führen. Auch das neue Konzept des ,,post-quota gender gaps“ verspricht einen analytischen Mehrwert für die Untersuchung der Wirkungsweise von Quoten, das in internationalen Vergleichen sehr gut eingesetzt werden kann. Wünschenswert wäre mehr Raum für den theoretischen Zusammenhang zwischen Wahlsystem und innerparteilichen Entscheidungsprozessen zur Kandidatinnenaufstellung gewesen. So bleiben die Mechanismen der Interaktion unklar und die Frage, warum Männern offenbar selbst unter günstigen Bedingungen höhere Listenplätze oder ,,sichere“ Wahlkreise angeboten werden, ist nicht abschließend geklärt. Zukünftige Forschung könnte die Rolle der Wählerinnen in das Modell einbeziehen, beispielsweise im Hinblick auf stereotype Zuschreibungen und Wahlverhalten. Insgesamt handelt es sich um eine sehr spannende und kenntnisreiche Studie und ist unbedingt Leserinnen mit Interesse an Wahl- und Parteienforschung empfohlen!

Funding Open Access funding enabled and organized by Projekt DEAL.

Open Access Dieser Artikel wird unter der Creative Commons Namensnennung 4.0 International Lizenz veröffentlicht, welche die Nutzung, Vervielfältigung, Bearbeitung, Verbreitung und Wiedergabe in jeglichem Medium und Format erlaubt, sofern Sie den/die ursprünglichen Autor(en) und die Quelle ordnungsgemäß nennen, einen Link zur Creative Commons Lizenz beifügen und angeben, ob Änderungen vorgenommen wurden.

Die in diesem Artikel enthaltenen Bilder und sonstiges Drittmaterial unterliegen ebenfalls der genannten Creative Commons Lizenz, sofern sich aus der Abbildungslegende nichts anderes ergibt. Sofern das betreffende Material nicht unter der genannten Creative Commons Lizenz steht und die betreffende Handlung nicht nach gesetzlichen Vorschriften erlaubt ist, ist für die oben aufgeführten Weiterverwendungen des Materials die Einwilligung des jeweiligen Rechteinhabers einzuholen.

Weitere Details zur Lizenz entnehmen Sie bitte der Lizenzinformation auf http://creativecommons.org/ licenses/by/4.0/deed.de. 